\title{
Hidden Manifestations of Inverse Polarity in Filaments
}

\author{
Boris P. Filippov \\ Institute of Terrestrial Magnetism, Ionosphere and Radio Wave \\ Propagation, Russian Academy of Sciences, Troitsk, Moscow Region, \\ 142092, Russia
}

\begin{abstract}
Some aspects of inverse polarity of transverse magnetic fields in filaments are presented following from their fine structure and structure of the chromosphere underneath.
\end{abstract}

At present magnetic field strength and direction in filaments can be only inferred from prominence (limb) field measurements. The complexity of measurements and ambiguity of interpretation hardly raise hopes that the magnetic configuration of filaments will be determined in the near future. Perhaps the fine structure of filaments in $\mathrm{H} \alpha$ and the arrangement of nearby chromospheric fibrils can tell us more about the magnetic configuration of filaments than direct measurements of magnetic fields therein, though the interpretation of these data is not a simple problem.

In $\mathrm{H} \alpha$ images filaments look like long thin threads running along a polarity inversion line. Threads are not infrequently twisted in ropes. The direction of threads reveals a direction of magnetic field which, hence, makes a small angle with the axis of the filament, in agreement with the field measurements in prominences. Though the transverse component of the filament field is less than the axial one, it is of great importance for the problem of filament equilibrium.

Martin et al. (1994) have studied in detail 150 filaments observed during 1989-1992 at Big Bear Solar Observatory. Their attention was concentrated basically on the field component directed along the axis of a filament but some conclusions from the results of their research can be made about the transverse component as well.

First, the plagette "tails" near a filament always tip away from the neutral line which is to say that the transverse field here is opposite to the large scale photospheric field. Second, Martin et al. (1994) pay attention to 'legs' or barbs of filaments. They divide all filaments into right-bearing and left-bearing. As the barbs represent bunches of threads extended from the main body of a filament, their orientation is specified by a direction of transverse field about the axial component. In so far as all dextral filaments have right-bearing barbs and sinistral filaments have left-bearing ones, both types are of inverse polarity. Lastly, the legs of filaments are never anchored in places of concentration of the background magnetic flux. In contrast, they avoid them.

To these facts it is possible to add one more following from the existence of structures noticed by us in the chromosphere (Figure 1) which look like fishbones with the symmetry axis parallel to the filament (Filippov 1995). Such structures arise near the boundary of the inversion zone where the transversal to the filament axis component changes its sign and only the component along 
a

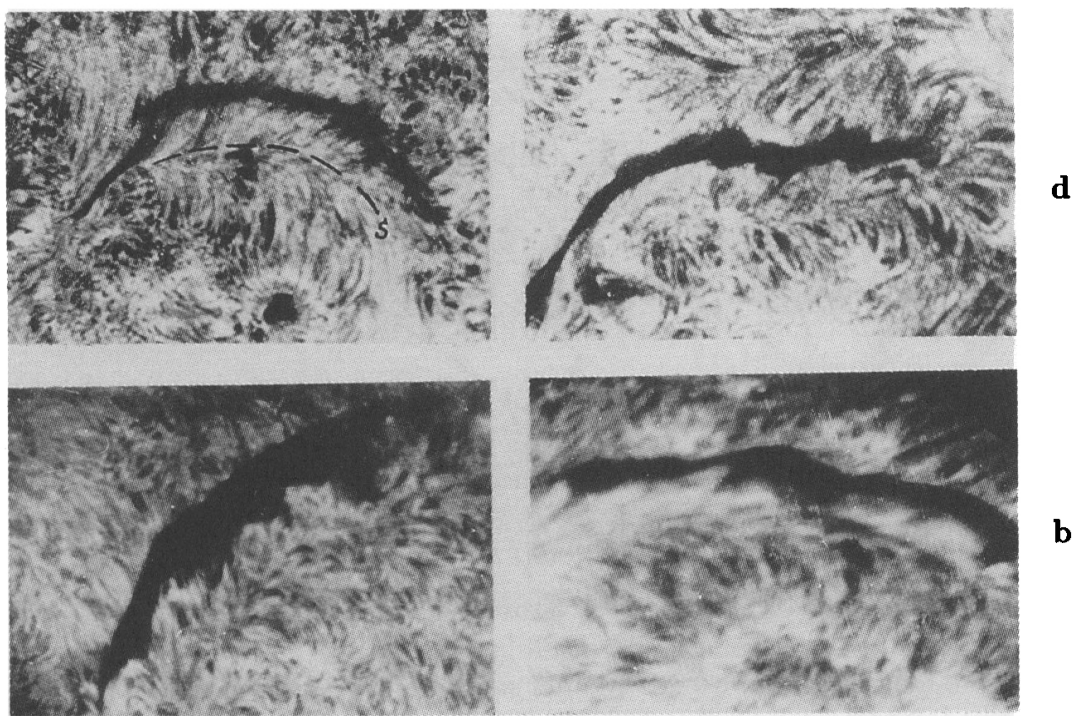

Figure 1. $\mathrm{H} \alpha$ filtergrams of the filament vicinities in the chromosphere. a) March 29, 1971 (courtesy of A. Bhatnagar), the dotted line $S$ is the axis of fish-bone structure, b) (courtesy of V. Makarov), c) June 18, 1989, d) June 11, 1980 (courtesy of V. Ishkov).

the filament remains on the boundary. The change of the direction of transverse field below a filament is the direct consequence of realization of equilibrium conditions in the model of inverse polarity.

Field lines of an inverse polarity filament should have a helical structure if the axial component is dominant. Many of the filaments actually look like well-defined helices. Everyone is familiar with the helical structure of eruptive prominences. There is the separatrix surface in the configuration of inverse polarity which separates the helical field lines of the filament current from the lines of the background field. The intersection of the separatrix surface with the chromosphere is the line $S$ (Figure 2).

It should be noted that in practice it may not be easy to determine whether an observed filament is a right-handed or left-handed helix. In fact, we observe only one projection of the real three-dimensional magnetic structure filled by matter very inhomogeneously. Depending on whether we see the top or bottom part of the right-handed helix, it reveals itself as threads deviated clockwise or counterclockwise from the axis of the filament. Rust and Kumar (1994) indicate that from the stability condition, at least for quiescent filaments, $\mathrm{H} \alpha$-absorbing material that makes the filament visible should rest on the underside of the helix where field lines have a U-like form. In eruptive filaments, opposite parts of a helix can display opposite Doppler shifts (Figure 3).

One of the arguments being put forward against inverse polarity models is the presumed absence of observed U-shaped structures in prominences. However, sometimes such structures are visible when a filament is activated and the helix is lifting and untwisting (see examples in Valniček et al. 1968, Webb et al. 1994, 


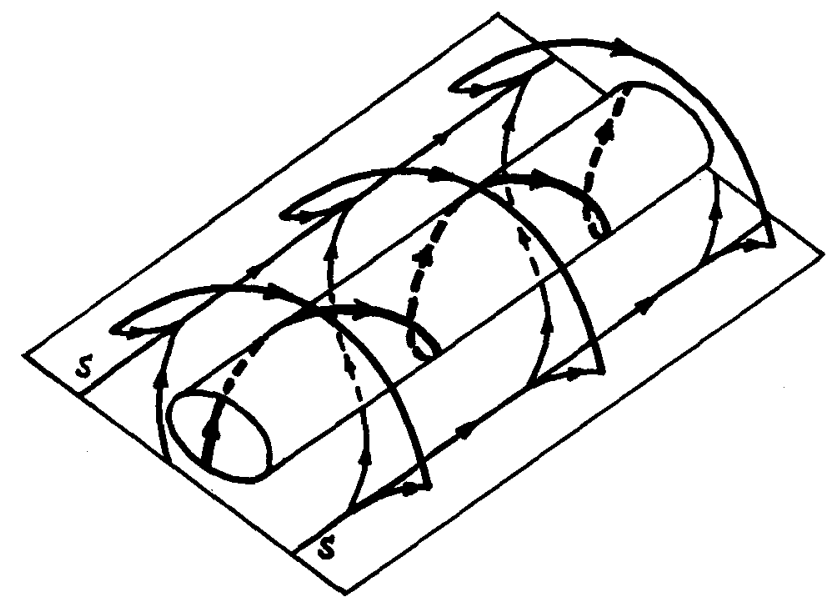

Figure 2. A sketch of the three-dimensional geometry of the inverse polarity magnetic configuration. Helical field lines of a filament screwing on a cylindrical surface are enclosed into the arch system of a background field. Integral lines of the tangential field in the chromosphere are shown by thinner lines, and the lines, $S$, are the fibril separatrices.
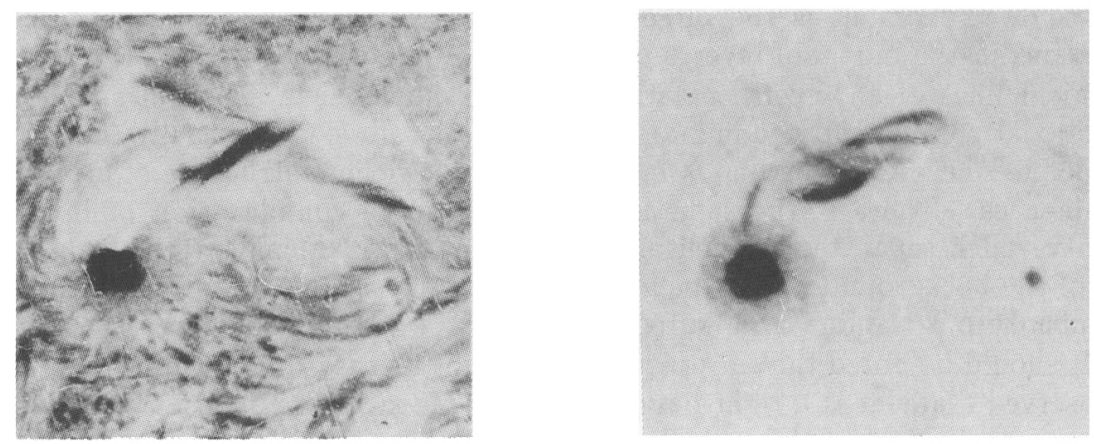

Figure 3. Filtergrams showing helical threads in the filament on June 27, 1980 06:20 UT. Left : $\mathrm{H} \alpha-0.5 \AA$, the threads are deflected counterclockwise to the axis of the filament; right: $\mathrm{H} \alpha+1.0 \AA$, the threads are deflected clockwise to the axis of the filament (courtesy of V. Ishkov). 


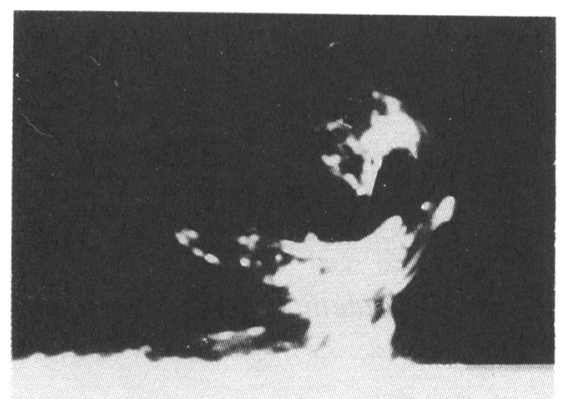

6:55 UT, $\mathrm{H} \alpha-1 \AA$

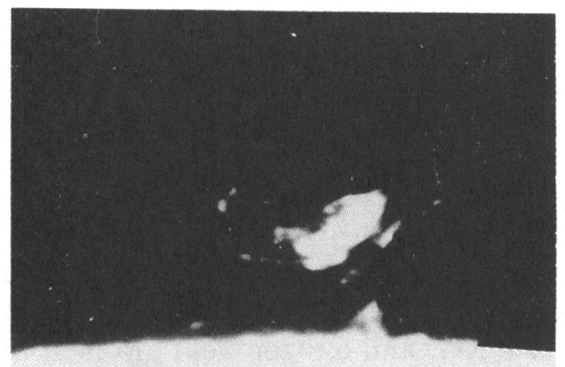

7:05 UT, H $\alpha-2 \AA$

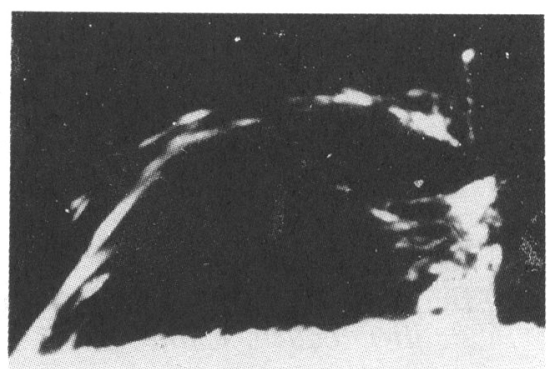

7:05 UT, $\mathbf{H} \alpha$

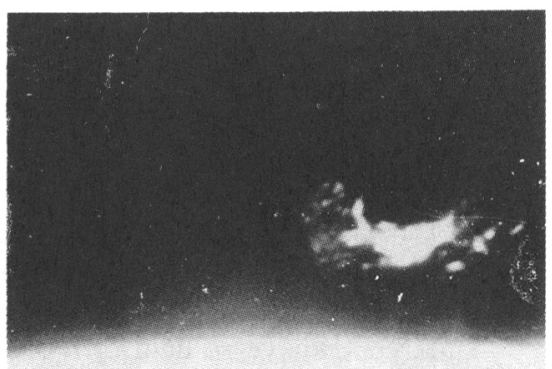

7:05 UT, H $\alpha-1 \AA$

Figure 4. U-shaped structures in a prominence on July 4, 1989 (courtesy of N. Shilova).

and in Figure 4). In quiescent prominences they are possibly shaded by the large-scale arch structure of a rope with anchored in the chromosphere ends.

Acknowledgments. This work was supported in part by the Russian Foundation for Basic Research under grants 96-02-16285, 96-02-18054 and Russian State Astronomical Program.

\section{References}

Filippov, B.P. 1995, A\&A, 303, 242

Martin, S.F., Bilimoria, R., and Tracadas, P.W. 1994, in Solar Surface Magnetism, (eds.) R.J. Rutten and C.J. Schrijver, New York: SpringerVerlag, p. 303

Rust, D.M. and Kumar, A. 1994, Solar Phys., 155, 69

Valniček, B., Godoli, G., and Mazzucconi, F. 1968, in Annals of the IQSY, A.C. Stickland, Cambridge, MA: The M.I.T. Press, p. 113

Webb, D.F. et al. 1994, Solar Phys., 153, 73 